\title{
Novel Battery Model of an All-Electric Personal Rapid Transit Vehicle to Determine State-of-Health through Subspace Parameter Estimation and a Kalman Estimator
}

\author{
C. R. Gould ${ }^{\dagger}$, C. M. Bingham, D. A. Stone, P. Bentley \\ UNIVERSITY OF SHEFFIELD \\ Department of Electronic and Electrical Engineering \\ Mappin Street, Sheffield, S1 3JD, UK \\ Tel. +44 (0) 114 2225847, Fax. +44 (0) 1142225196 , \\ †E-mail: c.gould@sheffield.ac.uk
}

\begin{abstract}
The paper describes a real-time adaptive battery model for use in an all-electric Personal Rapid Transit vehicle. Whilst traditionally, circuit-based models for lead-acid batteries centre on the well-known Randles' model, here the Randles' model is mapped to an equivalent circuit, demonstrating improved modelling capabilities and more accurate estimates of circuit parameters when used in Subspace parameter estimation techniques. Combined with Kalman Estimator algorithms, these techniques are demonstrated to correctly identify and converge on voltages associated with the battery State-of-Charge, overcoming problems such as SoC drift (incurred by coulomb-counting methods due to over-charging or ambient temperature fluctuations).

Online monitoring of the degradation of these estimated parameters allows battery ageing (State-of-Health) to be assessed and, in safety-critical systems, cell failure may be predicted in time to avoid inconvenience to passenger networks.

Due to the adaptive nature of the proposed methodology, this system can be implemented over a wide range of operating environments, applications and battery topologies.
\end{abstract}

Index Terms--Battery management systems; Energy storage; Parameter estimation; System identification.

\section{INTRODUCTION}

The ULTra (Urban Light Transport - Fig. 1) is a Personal Rapid Transit (PRT) vehicle developed by Advanced Transport Systems Ltd [1], which is an unmanned, steer-by-wire electric vehicle capable of carrying a $500 \mathrm{~kg}$ payload at speeds up to $40 \mathrm{kmph}$, for the purpose of improving the transportation needs of a busy city or other public space (e.g. Heathrow airport in this case) with minimal environmental impact.

With the increasing requirements for environmentallyfriendly vehicles, and the search for adequate traffic congestion solutions, more and more cities are turning to small all-electric or hybrid-electric passenger vehicles, promoting greater research into the requirements of

The authors would like to thank the Engineering and Physical Sciences Research Council for supporting this work. efficient energy storage systems and their management.

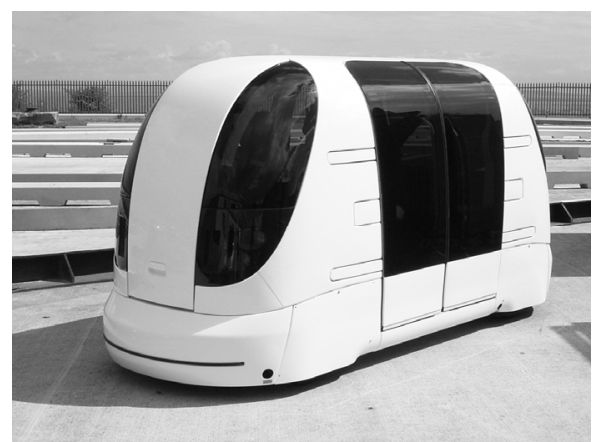

Fig. 1.ULTra Personal Rapid Transit vehicle

Moreover, with the increasing development of X-bywire control systems, the large transient power demands (Fig. 2) on such vehicles are requiring more accurate battery State-of-Function $(\mathrm{SoF})$ prediction techniques, so that State-of-Charge (SoC - the amount of charge available in the battery at any time to sink/source excessive transients) and State-of-Health ( $\mathrm{SoH}$ - the ability of that battery to repeatedly provide its rated capacity over time), can be readily assessed to avoid inconvenience to passengers and/or catastrophic failure of the safety-critical battery pack.

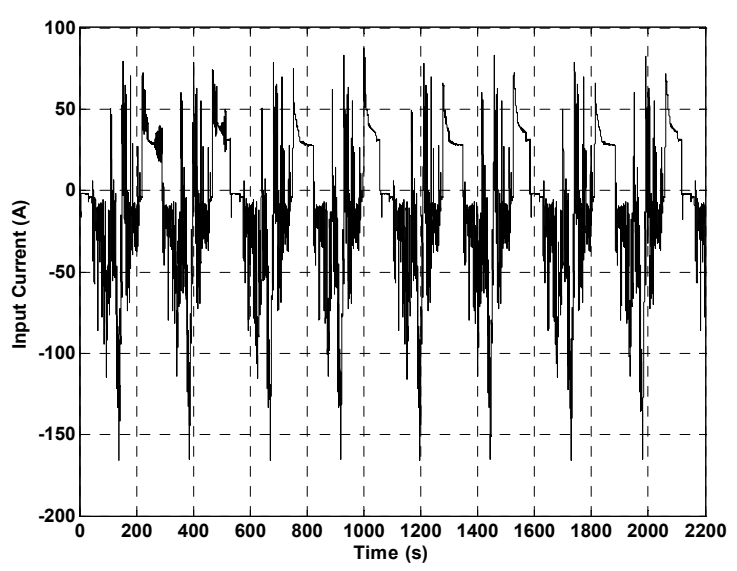

Fig. 2. ULTra driving cycle current-profile 
Whilst SoC characterisation techniques have been published for lead-acid battery technologies [2-6], SoH determination is less well-reported, primarily because the $\mathrm{SoH}$ is a qualitative rather than quantitative measure of the ageing of a battery $[7,8]$. In most cases, the $\mathrm{SoH}$ is estimated by monitoring the degradation of specific battery model parameters, as suggested in [2], where $\mathrm{SoH}$ deterioration is demonstrated through use of the wellknown Randles' battery circuit model [9] using static parameters in a closed-loop Extended Kalman Filter (EKF). For dynamic drive cycles described by Fig. 2, however, static parameter values will not suffice to produce an accurate model in either open- or closed-loop systems.

This paper focuses on providing an accurate dynamic model of systems placed under stresses such as the ULTra drive-cycle, with its large bipolar transients and requirements for occasional charge scenarios. Here it will be shown that the Randles' model can be mapped to an equivalent circuit, allowing Subspace parameter estimation algorithms to be performed online in combination with a simple Kalman Estimator (KE). This model is demonstrated to be more accurate than the Randles' circuit for estimation algorithms, allowing adaptive identification and convergence of the model voltages associated with $\mathrm{SoC}$ and $\mathrm{SoH}$ monitoring. The resulting dynamic parameter estimates will also be shown to be useful for fault-prediction and battery end-of-life determination.

\section{BATTERY MODEL}

The ULTra relies on a system of $12 \mathrm{~V}$ Valve-Regulated Lead-Acid (VRLA) batteries, nominally rated between 45Ahrs (at the $\mathrm{C} 5$ rate) and 50Ahrs (at the $\mathrm{C} 20$ rate).

Fig. 3 illustrates the Randles' model circuit where $R_{d}$ represents the self-discharge resistance, $C_{b}$ is considered the main charge store, and the voltage across it a suitable indicator of SoC, whilst $\mathrm{SoH}$ can be inferred by any decrease in the value of $C_{b} . R_{i}$ models the resistance of the battery's terminals and inter-cell connections, whilst $R_{t}$ and $C_{s}$ describe transient effects resulting from shifting ion concentrations and plate current densities.

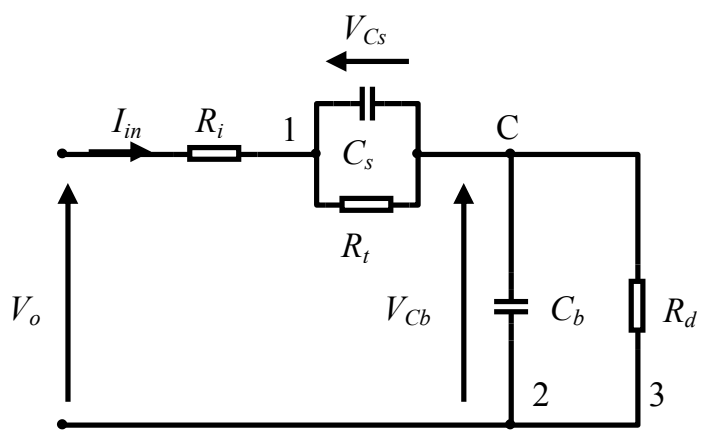

Fig. 3. Randles' battery model

Through use of a star-delta transform, the Randles' model may be mapped to the equivalent circuit shown in Fig. 4, where the star points of Fig 3 are labelled 1, 2 and
3 , with centre C. Note that points 2 and 3 are shorted, and therefore the resulting delta points will also require a short in parallel with the impedance expected.

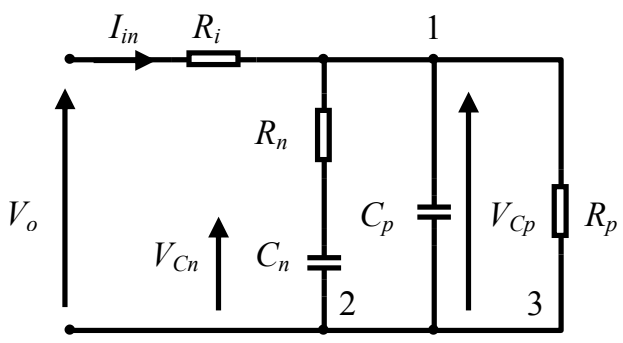

Fig. 4. Mapped equivalent circuit

The remaining two impedances are manipulated until sensible circuit parameters are achieved, resulting in an impedance consisting of a capacitor and resistor in series $\left(C_{n}\right.$ and $\left.R_{n}\right)$, and an impedance consisting of a capacitor and resistor in parallel $\left(C_{p}\right.$ and $\left.R_{p}\right)$. The component mappings and SoC voltage mapping is shown in (1) and (2) respectively, whilst state-space equations are listed in (3) for Randles', and (4) for the mapped circuit.

$$
\begin{gathered}
C_{n}=C_{b}^{2} /\left(C_{b}+C_{s}\right) \quad C_{p}=C_{b} C_{s} /\left(C_{b}+C_{s}\right) \\
R_{n}=R_{t}\left(C_{b}+C_{s}\right)^{2} / C_{b}^{2} \quad R_{p}=R_{d}+R_{t} \\
V_{C_{b}}=\left(V_{C_{n}} C_{n}+V_{C_{p}} C_{p}\right) /\left(C_{n}+C_{p}\right) \\
\dot{V}_{C_{b}}=\left(I_{i n} R_{d}-V_{C_{b}}\right) / C_{b} R_{d} \\
\dot{V}_{C_{s}}=\left(I_{i n} R_{t}-V_{C_{s}}\right) / C_{s} R_{t} \\
V_{o}=V_{C_{s}}+V_{C_{b}}+I_{i n} R_{i} \\
\dot{V}_{C_{n}}=-\frac{V_{C_{n}}}{R_{n} C_{n}}+\frac{V_{C_{p}}}{C_{n} R_{n}} \\
\dot{V}_{C_{p}}=\frac{V_{C_{n}}}{R_{n} C_{p}}-\frac{V_{C_{p}}\left(R_{n}+R_{p}\right)}{C_{p} R_{n} R_{p}}+\frac{I_{i n}}{C_{p}} \\
V_{o}=V_{C_{p}}+I_{i n} R_{i}
\end{gathered}
$$

\section{SUBSPACE PARAMETER ESTIMATION:}

The input-output relationship of a discrete-time state space model with system matrices $A_{d}, B_{d}, C_{d}$ and $D_{d}$ and white noises $w(t), v(t)(5)$, can also be represented by the relationship shown in (6), for any invertible matrix ' $T$ '. It can be shown (Ljung 10.80-10.129 [10]), that the system of matrices $A_{d}, B_{d}, C_{d}, D_{d}$, and initial conditions $\tilde{x}(0)$ given sufficient input-output data, may then be estimated by solution of the resulting Subspace algorithms by a least squares method.

$$
\begin{aligned}
& x(t+1)=\mathrm{A}_{\mathrm{d}} x(t)+\mathrm{B}_{\mathrm{d}} u(t)+w(t) \\
& y(t)=\mathrm{C}_{\mathrm{d}} x(t)+\mathrm{D}_{\mathrm{d}} u(t)+v(t)
\end{aligned}
$$




$$
\begin{aligned}
& \tilde{x}(t+1)=T^{-1} \mathrm{~A}_{\mathrm{d}} T \tilde{x}(t)+T^{-1} \mathrm{~B}_{\mathrm{d}} u(t)+\widetilde{w}(t) \\
& y(t)=\mathrm{C}_{\mathrm{d}} T \tilde{x}(t)+\mathrm{D}_{\mathrm{d}} u(t)+v(t)
\end{aligned}
$$

This allows static battery model parameters to be estimated for a 'window' of input-output data $\left(I_{\text {in }}\right.$ and $\left.V_{o}\right)$. To capture the dynamic variation of the battery parameters, a FIFO buffer is used to store the accumulation of input-output data. Due to the large timeconstants involved with $C_{n}$ and $R_{p}$ it is impossible to acquire sufficient buffered data for accurate estimation of the exact values of $C_{n}$ and $R_{p}$, without neglecting other, more transient parameter variations (i.e. occasional charging periods), and so a structured Subspace estimation model is used in which the time constant associated with the product $C_{n} R_{n}$ is assumed to be large (compared to the time constant associated with $C_{p} R_{n}$ ) and constant, over a small buffer of data ( 90 seconds). Likewise, $R_{p}$ is assumed to be sufficiently large $(5 \mathrm{k} \Omega)$ so that $\left(R_{n}+R_{p}\right) / R_{p} \rightarrow 1$. Inaccuracies in $C_{n}$ will be corrected for by the inherent $\mathrm{KE}$ predictor-corrector action, allowing determination of $C_{n}$, and hence $C_{b}$ and SoH.

\section{KALMAN ESTIMATOR THEORY}

The Kalman Estimator [11] is particularly useful for this methodology, since it optimally estimates states affected by broadband noise within the system bandwidth (i.e. tries to minimise the sum-of-squared errors between actual and estimated states), which may not be able to be filtered classically. The recursive use of predictorcorrector algorithms is also beneficial, since at each stage, the updated parameter estimates may be used to aid the $\mathrm{KE}$ in convergence.

The implementation of the KE is similar in all respects to [2], whereby a discrete-time equivalent model (as used in the Subspace parameter estimation) of a system's statevariable description (7) is generated, using a first-order Taylor series expansion, resulting in (8).

$$
\begin{aligned}
\dot{x}(t) & =\mathrm{A} x(t)+\mathrm{B} u(t) \\
y(t) & =\mathrm{C} x(t) \\
x_{k+1} & =\mathrm{A}_{\mathrm{d}} x_{k}+\mathrm{B}_{\mathrm{d}} u_{k} \\
y_{k+1} & =\mathrm{C}_{\mathrm{d}} x_{k+1}
\end{aligned}
$$

where, with sampling period, $T_{c}$

$$
\mathrm{A}_{\mathrm{d}} \approx \mathrm{I}+\mathrm{A} T_{c} \quad \mathrm{~B}_{\mathrm{d}} \approx \mathrm{B} T_{c} \quad \mathrm{C}_{\mathrm{d}} \approx \mathrm{C}
$$

This system is now assumed to be corrupted by stationary Gaussian white noise consisting of a system disturbance additive vector $\sigma_{k}$ and model disturbance additive vector $\mu_{k}$, both of which are considered to have zero mean value for all $k$ and have the following respective covariance matrices

$$
\begin{array}{ll}
\mathrm{E}\left[\sigma_{k} \sigma_{k}{ }^{\mathrm{T}}\right]=Q & \text { for all } k \\
\mathrm{E}\left[\mu_{k} \mu_{k}^{\mathrm{T}}\right]=R & \text { for all } k
\end{array}
$$

where $\mathrm{E}$ is the expectation operator. (11)

This allows the physical system to be described by

$$
\begin{aligned}
& x_{k+1}=\mathrm{A}_{\mathrm{d}} x_{k}+\mathrm{B}_{\mathrm{d}} u_{k}+\Gamma \sigma_{k} \\
& z_{k+1}=\mathrm{C}_{\mathrm{d}} x_{k+1}+\mu_{k+1}
\end{aligned}
$$

where $z$ is now used to describe the measured output (e.g. $V_{o}$ incorporating the Gaussian noise) and $\Gamma$ represents the coupling between the model disturbances on each state.

Beneficially, the KE may be implemented recursively, so that at each sample step ' $k$ ' the algorithms operate on input and output data collected up to and including the $k^{\text {th }}$ sample in a prediction/correction system as demonstrated in the diagram shown in Fig 5. Intuitively this will require initial estimates for the states and covariance matrices considered by the model.

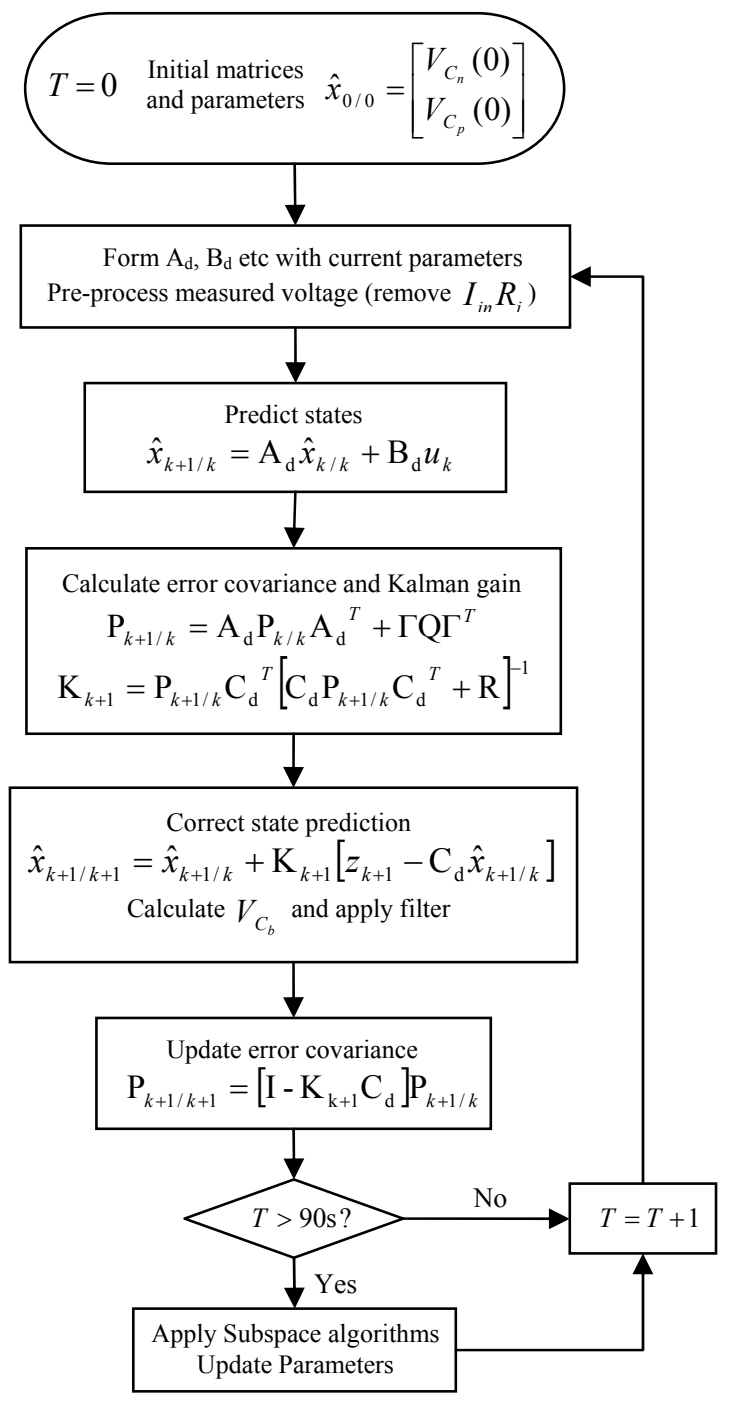

Fig. 5. Recursive implementation of KE algorithms 
With respect to notation, the $\mathrm{KE}$ estimates are described as in [2], where the KE estimate $\hat{x}_{k+1 / k+1}$ is calculated from the previous estimate $\hat{x}_{k / k}$, the input signals (in this case current into the positive terminal of the battery) is termed $u$, and the measured signals, $z$.

The KE is implemented using the state-space equations of (4), and initial conditions and covariance matrices of (12), whilst the sampling frequency is selected to be $1 \mathrm{~Hz}$ to maintain model accuracy whilst avoiding excessive computation complexity. The sample time, is therefore selected to be $T_{c}=1 \mathrm{~s}$.

The output voltage is first pre-processed to remove the voltage dropped across $R_{i}$ (known from parameter estimation), allowing one of the states $\left(V_{C_{p}}\right)$ to be immediately calculated. This allows greater prediction accuracy over the Randles' model.

$$
\begin{aligned}
& P_{0}=\left[\begin{array}{cc}
10 & 0 \\
0 & 10
\end{array}\right] \quad Q=\left[\begin{array}{cc}
0.01 & 0 \\
0 & 0.1
\end{array}\right] \\
& R=10 \quad x_{0 / 0}=\left[\begin{array}{l}
13.3 \\
13.3
\end{array}\right]
\end{aligned}
$$

\section{RESULTS}

The KE is initialised with $R_{i}=6 \mathrm{~m} \Omega, R_{n}=10 \mathrm{~m} \Omega, C_{p}=$ 2000F, $C_{n}=90000 \mathrm{~F}$, and used to monitor the effect that a continuous series of cycles constructed from Fig. 2 has on a fully charged, well-conditioned battery. After 90s, and at regular subsequent intervals (10s in this case) the Subspace algorithms of [10] are used to adapt the battery model parameters online, resulting in the time-varying parameters shown in Fig. 6, 7 and 8, demonstrating that careful monitoring of these parameters can indicate dangerous areas of operation such as overcharge/discharge situations as well as possible fault conditions such as cell short-circuits.

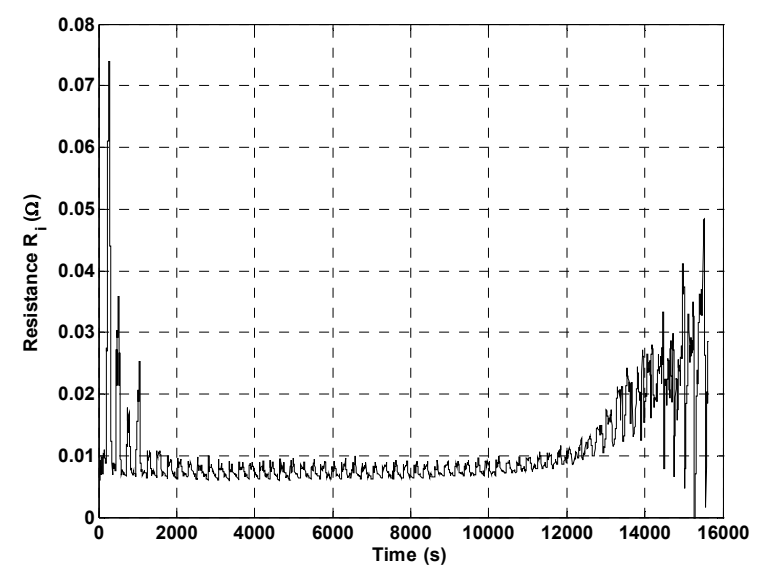

Fig. 6. Online estimated battery parameter: $R_{i}$

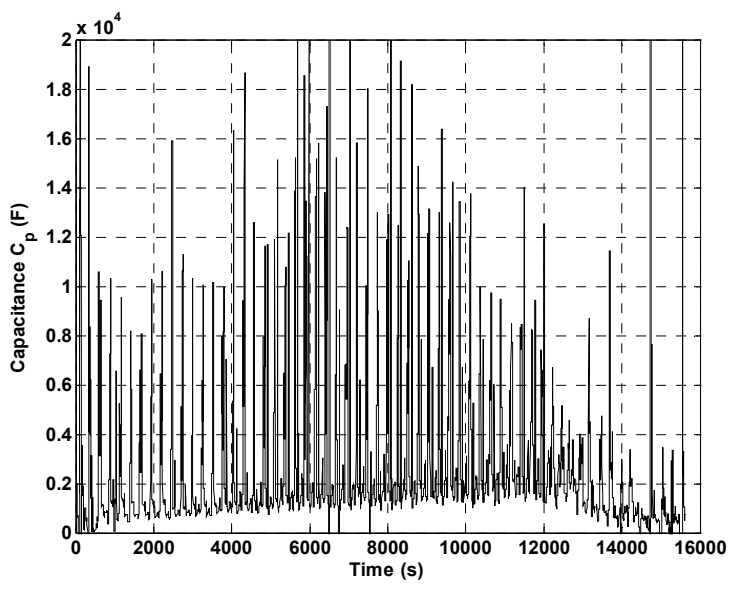

Fig. 7. Online estimated battery parameter: $C_{p}$

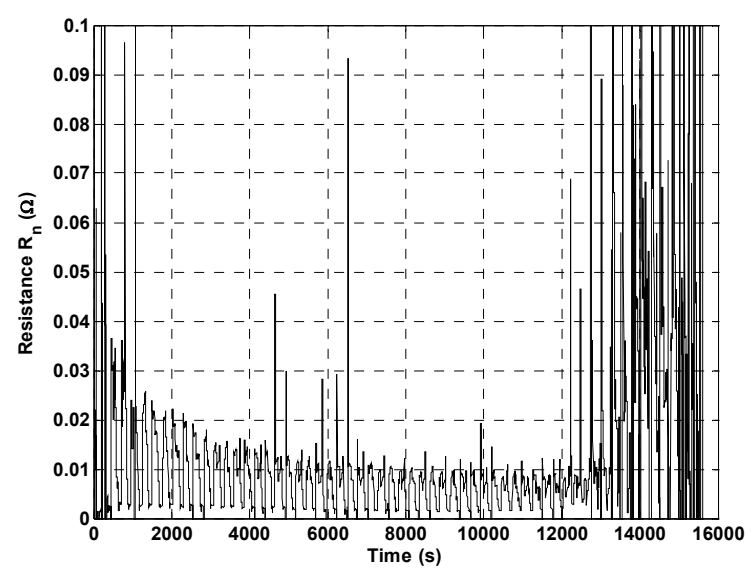

Fig. 8. Online estimated battery parameter: $R_{n}$

The KE estimates of $V_{C_{n}}$ and $V_{C_{p}}$ can be manipulated using (2) to provide an estimate of $V_{C_{b}}$ (indicating the variation of $\mathrm{SoC}$ ), which is passed through a low-pass filter $\left(\omega_{c}=0.057 \mathrm{rads}^{-1}\right)$, and shown in Fig. 9, to demonstrate the ability of the KE to converge compared to a current integration technique (which requires a priori knowledge of $C_{b}$ and $\left.V_{C_{b}}(0)\right)$.

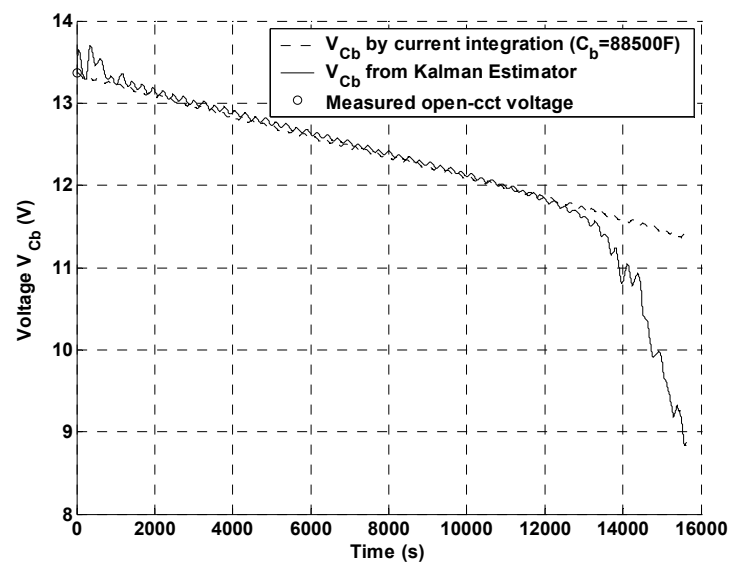

Fig.9 Estimated $V_{C b}$ showing convergence of KE to correct initia condition using mapped circuit model 


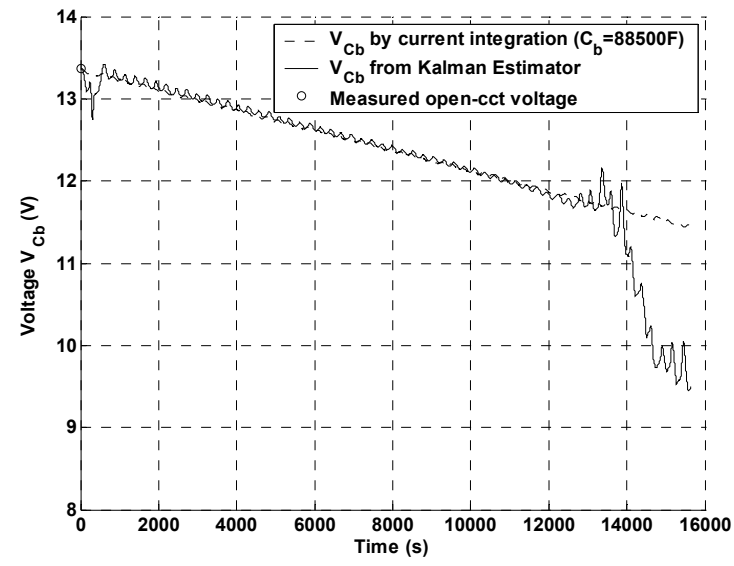

Fig.10 Estimated $V_{C b}$ using Randles' circuit model showing incorrect parameter estimation and convergence for Time $>12000 \mathrm{~s}$

Fig.10 demonstrates the result if the Randles' model is used instead of the mapped model for both Subspace parameter estimation and the $\mathrm{KE}$ algorithms. It can be seen that both models exhibit excellent initial convergence, yet it is evident that the Randles' model shows incorrect convergence due to erroneous parameter estimation, for operation at low SoC (i.e. after 12000s). Conversely, the advantageous feature of the mapped equivalent circuit's readily identifiable state allows more accurate parameter estimation, and subsequently better voltage convergence.

The variation in $C_{b} / \mathrm{SoH}$ (Fig. 11) may be estimated by applying a linear curve-fit to buffered estimations of $V_{C_{b}}$, and by considering the integrated current through this capacitor over these buffer durations (typically 1800s to provide stable results).

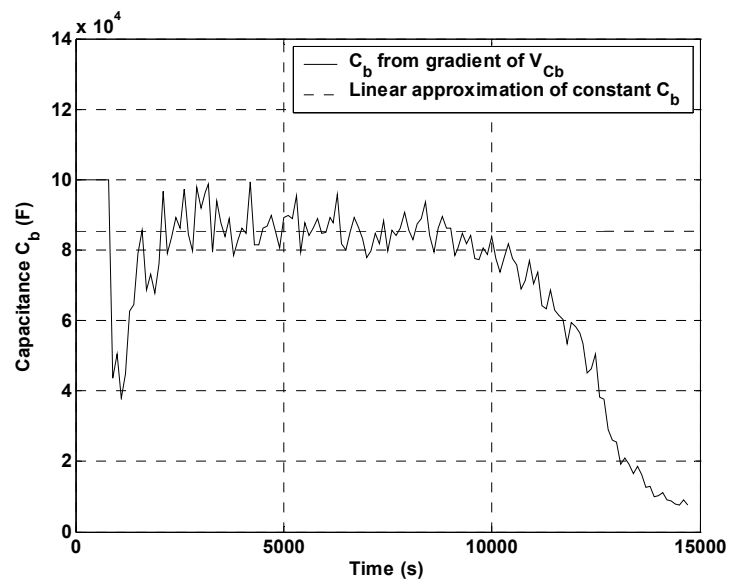

Fig. 11 Resulting variation of $C_{b}$ from gradient of observed $V_{C b}$ of Fig. 9

It can be seen that after the initial convergence of the Subspace parameter and KE algorithms, the value of $C_{b}$ settles to an almost constant average value between $3000 \mathrm{~s}$ and 10000 s (i.e. the region of preferred operation between $\sim 80 \%$ and $\sim 30 \%$ SoC). This action can be used during accelerated ageing tests, where the battery is charged at the $\mathrm{C} 10$ rate, and then discharged by a continuous sequence of the current profile illustrated in Fig. 2, until catastrophic battery failure is witnessed. The resulting degradation of the average value of $C_{b}$ can then be seen over time (Fig. 12), and a linear relationship applied (13), where $N$ is the test number.

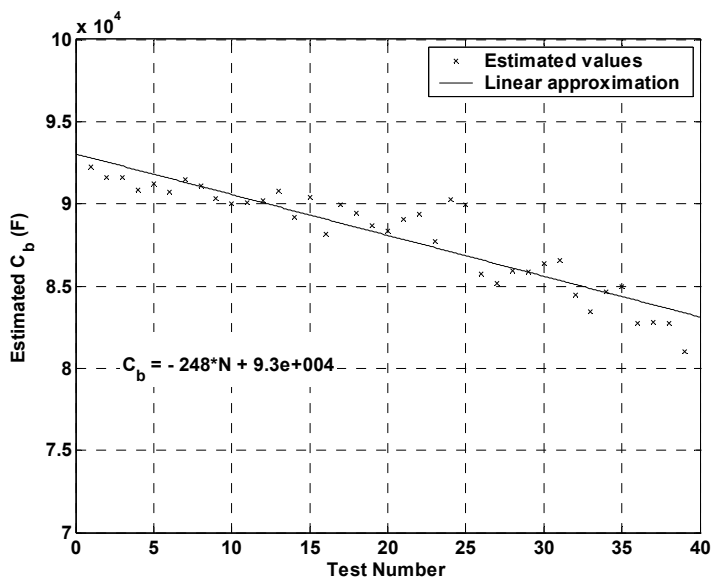

Fig. 12. Decline in estimated $C_{b}$ with 'age' of battery

$$
C_{b}=-248 N+93000
$$

In a similar manner, as each test is implemented, the total current demand can be integrated, allowing an estimate of the total available capacity of the battery during each test. The reduction in the available capacity of the battery under test can be seen in Fig. 13, and again, this relationship may be approximated with the linear expression shown in (14).

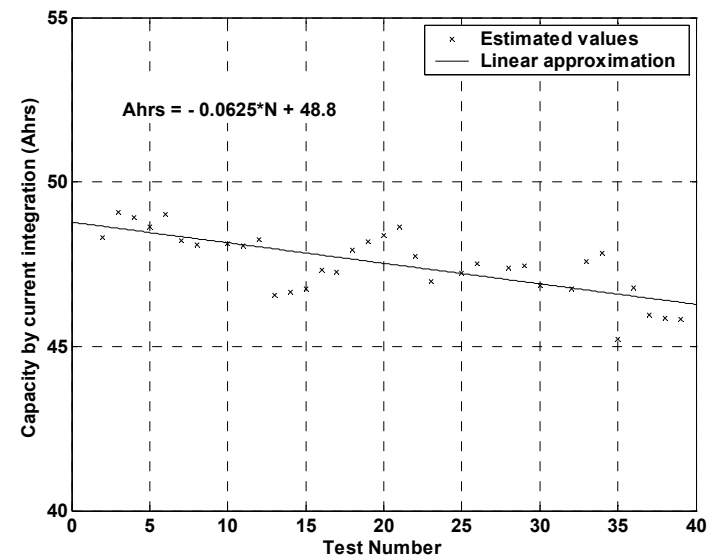

Fig. 13. Reduction of available capacity with 'age' of battery

$$
\text { Ahrs }=-0.0625 N+48.8
$$

By manipulation of (13) and (14), an expression (15) relating the total available capacity of a battery under test to the estimated average value of $C_{b}$ can be found (See Fig. 14), so that not only the relative State-of-Health can be estimated, but also the total capacity of the battery at that particular time. This in turn allows more accurate current-integration SoC techniques to be employed (now that the necessary a priori knowledge can be estimated), and corrected using the convergence features of the KE 
algorithms to ensure correct $V_{C_{b}}$ and State-of-Charge estimation is achieved.

$$
\text { Ahrs }=0.00025 C_{b}+25.4
$$

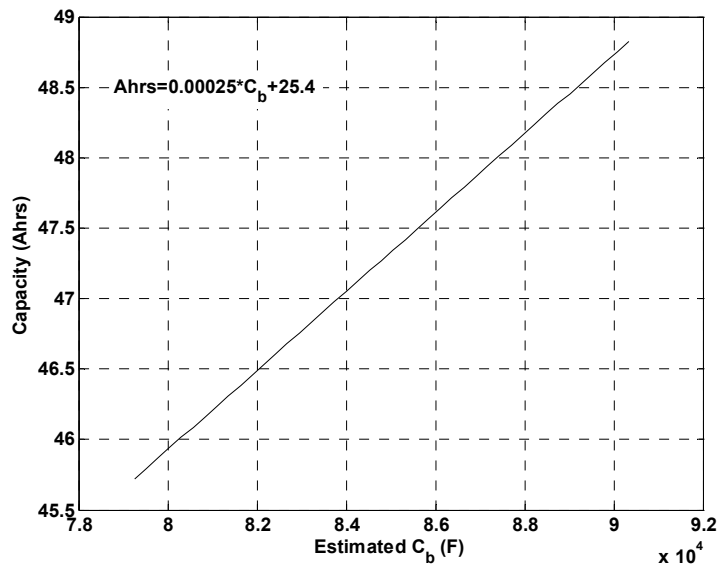

Fig. 14. Relationship between estimated $C_{b}$ and available capacity

As an example, for the case of the battery with $\mathrm{SoH}$ and $\mathrm{SoC}$ demonstrated in Fig 9, the estimated $C_{b}$ of $88500 \mathrm{~F}$ from Fig 11 maps to an available capacity of 47.5Ahrs, compared to that of 47.4Ahrs achieved by integrating the total current demand. The battery is therefore shown to be in good health.

\section{CONCLUSION}

A novel battery modelling methodology for an allelectric Personal Rapid Transit vehicle is proposed consisting of novel battery circuit model, Kalman Estimator and novel online Subspace parameter estimation, for the purpose of battery State-ofHealth/State-of-Charge estimation. The use of Subspace estimation algorithms allows the observer to adaptively estimate and converge on voltages associated with these functionality indicators, so that ambient temperature effects and battery self-discharge issues are automatically compensated for. Results show improved voltage predictions compared to algorithms including the Randles' model. Moreover the resulting parameter estimations can identify fault conditions and areas of unsafe operation.

\section{ACKNOWLEDGMENT}

The authors would like to thank Advanced Transport Systems Ltd for their cooperation in this work, and particularly Nicolas Zurlinden for providing the ULTra driving cycle data and press-shots.

\section{REFERENCES}

[1] http://www.atsltd.co.uk

[2] B. S. Bhangu, P. Bentley, D. A. Stone, C. M. Bingham, "Observer techniques for estimating the state-of-charge and state-of-health of VRLABs for hybrid-electric vehicles," Vehicle Power and Propulsion, 2005 IEEE Conference, p.780-789, Sep. 2005

[3] Sinclair, R. Duke, S. Round, "An adaptive battery monitoring system for an electric vehicle," Proceedings. International Conference on Power Electronic Drives and Energy Systems for Industrial Growth, vol.2, p786-791, Dec. 1998

[4] C. S. C. Bose, F. C. Laman, "Battery state of health estimation through coup de fouet," 22nd International Telecommunications Energy Conference (INTELEC), p597-601, 2000

[5] Atsuo Kawamura, Takahiro Yanagihara, "State of charge estimation of sealed lead-acid batteries used for electric vehicles", 29th Annual IEEE Power Electronics Specialists Conference, vol.1, p583-587, May 1998

[6] Pyung-Soo Kim, "New estimation filtering for battery management systems of lead-acid cells in hybrid electric vehicles", Int. Journal of Computer Science and Network Security, vol.7, no.2, Feb. 2007

[7] D. Berndt, E. Meissner, R. Rusch, "Aging effects in valveregulated lead-acid batteries", 15th International Telecommunications Energy Conference (INTELEC), vol.2, p139-145, Sep. 1993

[8] P. Ruetschi, "Aging mechanisms and service life of leadacid batteries", Journal of power souces, vol.127, p33-44, 2004

[9] K. J. Vetter, "Elecktrochemische Kinetik", Springer, Berlin, p268, 1961

[10] L.Ljung, "System identification - Theory for the user", 2nd ed, PTR Prentice Hall, p317-353, 1999

[11] R. E. Kalman, "A new approach to linear filtering and prediction problems", Transactions of the ASME - Journal of Basic Engineering, Series D, p.35-45, 1982 